\title{
Test Facility Country
}

National Cancer Institute

\section{Source}

National Cancer Institute. Test Facility Country. NCI Thesaurus. Code C90467.

The country in which the test is conducted. 